\title{
CHARACTERIZATION OF A RAT MODEL OF INSULIN DEFICIENCY INDUCED VASCULAR COMPLICATION
}

\author{
Nora Desoky,Hany M. El-Bassossy*\& Ahmed Fahmy \\ Department of Pharmacology, Faculty of Pharmacy, Zagazig University, Egypt \\ Department of Pharmacology, Faculty of Pharmacy, King Abdulaziz University, Kingdom of Saudi Arabia*
}

\begin{abstract}
Vascular dysfunction is one of the important diabetic complications. Here we fully characterized a rat model of streptozotocin (STZ) induced vascular complication.

Diabetes was induced by single intraperitoneally injection of STZ (50 mg. $\mathrm{kg}^{-1}$, ip).Blood pressure (BP) and isolated aorta responses phenylephrine (PE), KCl, acetylcholine (ACh), sodium nitroprusside (SNP) were recorded after 6, 8 and 10 weeks of STZ injection.In addition, serum levels of glucose, tumor necrosis factor $\alpha$ (TNF $\alpha$ ), lipids, advancedglycation end products (AGEs), and arginase activity were determined. Furthermore, aortic reactive oxygen species(ROS) generation, hemeoxygenase-1 expression and collagen deposition were examined.

Streptozotocin injection resulted in a significant hyperglycemia after 3 days of injection which was stable for 10 weeks. Diabetes was associated with a significant increasein BP after 6 weeks which was stable at 8 weeks. Aorta isolated from diabetic animals showed exaggerated contractility to $\mathrm{PE}$ and KCland impaired relaxation to ACh compared to control after 6 weeks which were clearer at 8 weeks of STZ injection. In addition, diabetic animals showed significant increases in serum levels of lipids, AGEs, TNF $\alpha$ and arginase enzyme activity after 8 weeks of STZ compared to control. Furthermore, aortae isolated from diabetic animals were characterized by increased ROS generation and collagen deposition.

In conclusion, injecting rats with STZ at dose $50 \mathrm{mg} \cdot \mathrm{kg}^{-1}$ produces a model of diabetic vascular complication after 8 weeks that are characterized by hypertension, disturbed vascular reactivity, elevated serum lipids, inflammatory cytokines and enzymes and enhanced aortic ROS generation and collagen deposition.

Key words: Diabetes; aorta; hypertension; vascular reactivity, rat

Abbreviations: ANOVA, analysis of variance; NO, nitric oxide; PE, phenylephrine; ROS, reactive oxygen species; SNP, sodium nitroprusside;Ach, acetyl choline; BP, blood pressure.
\end{abstract}

\section{INTRODUCTION}

D iabetes mellitus is a complex, multisystem disease that representsthe most common metabolic disorder. Many of the complications of diabetes are vascular in origin, and this puts diabeticsat an increased risk of cardiovascular diseases [1].Human diabetics have a markedly increased morbidity and mortality due to cardiovascular disease[2;3].

Severalfactors have been reported to explain the vascular dysfunction and acceleratedatherosclerosis in diabetes, including hyperglycemia itself, production of advanced glycation end products (AGEs), hyperlipidemia and hypertension. Increased oxidative stress is particularly of greatimportance as a pathogenic factor of diabetesassociatedvascular disease [4;5].Changes in vascular responsiveness to vasoconstrictors and vasodilatorsare mainly responsible for development of some vascularcomplications of diabetics[6]. Most of thesecomplications are due to augmentedgeneration of reactive oxygen species (ROS) which lead to endotheliumdysfunction [7] In spite of the significant developments in antidiabetic therapy, diabetic complications continue to be seriously deleterious. Many of the clinical complications of diabetes may be ascribed to alterations in vascular structure and function, with subsequent end-organ damage and death [8].
Therefore, preventing vascular complications in diabetes can save diabetic patient from other complications.

New drugs are continually being tested and new strategies developed to prevent and treat diabetes. In thesestudies, different experimental animal models of diabeteshave been used. One of the most important diabetes models is streptozotocin (STZ)induced diabetes in the rat[9]. However, little is known about the onset of developing vascular complications and their characteristics. The aim of the present work was to fully characterize the rat streptozotocin model in order to provide supporting information for researchers who wish to work on diabetic vascular complications.

\section{Materials and methods}

\subsection{Animals}

Adult male Wistar rats weighing 140-160 g (Zagazig University, Zagazig, Egypt) were housed in clear polypropylene cages (4rats per cage) and kept on a light-dark cycle of equal duration, under constant environmental conditions. Rats were fed commercially available rat normal pellet diet and water ad libitum. Experimental design and animal handling were according to the guidelines of the Ethical Committee of the Faculty of Pharmacy, Zagazig University, for Animal Use.

2.2 Study protocol 
Animals were randomly divided into four experimental groups (6 animals each); control, diabetic (6 weeks),diabetic ( 8 weeks), diabetic (10 weeks). Diabetes was induced by a single intraperitoneal injection of streptozotocin (STZ, 50 $\left.\mathrm{mg} \cdot \mathrm{kg}^{-1}\right)$.

At the end of the study, blood pressure was measured and rats were anesthetized with diethyl ether. Blood was collected from the retro-orbital plexus and centrifuged $\left(3000 \times \mathrm{g}, 4^{\circ} \mathrm{C}, 20 \mathrm{~min}\right)$ to separate serum that was analysed for glucose, insulin, tumour necrosis factor $\alpha$ (TNF $\alpha)$, cholesterol, low density lipoprotein cholesterol (LDL-C), advanced glycation end products (AGEs) and arginase activity. Then, through opening the abdomen, descending thoracic aorta was carefully excised and placed in a Petri dish filled with cold Krebs-Henseleit buffer containing (in $\mathrm{mM}$ ): $\mathrm{NaCl}$ 118.1, $\mathrm{KCl}$ 4.69, $\mathrm{KH}_{2} \mathrm{PO}_{4}$ 1.2, $\mathrm{NaHCO}_{3}$ 25.0, glucose 11.7, $\mathrm{MgSO}_{4} 0.5$ and $\mathrm{CaCl}_{2} 2.5$. The aorta was cleaned of excess connective tissue and fat and cut into rings of approximately $3 \mathrm{~mm}$ in length. For each animal, one aortic ring was suspended in an organ bath for studying vascular reactivity while the second was snap frozen until measurement of reactive oxygen species (ROS) generation. The third rapidly stored in $10 \%$ neutral formalin solution for examining aortic collagen deposition andhemeoxygenase-1 expression.

\subsection{Serum analysis}

Serum glucose was determined using blood glucose meter system (one touch brand, U.S.A), according to the method[10].

Serum arginase activity was measured as previously described [11] using arginase assay kit ${ }^{\circ}$ (Biodiagnostic, Cairo, Egypt). The method depends upon the colorimetric determination of the arginase enzyme product, urea. The serum was incubated for 1 hour withreaction mixture containing the activator (manganesesulphate) and substrate (arginine) in carbonate buffer $(\mathrm{pH}$ 9.5). The produced urea was condensed with diacetylmonoxime in an acid medium in the presence of ferric chloride (oxidant) and carbazide (accelerator). Then the absorbance was measured at $525 \mathrm{~nm}$.

Serum AGEs was determined as previously described[12;13]. In brief, serum was diluted 1:15 in saline and the fluorescence intensity at $(\lambda \mathrm{ex}=$ 370 or $440 \mathrm{~nm})$ was determined by LS45 fluorescence spectrophotometer (PerkinElmer®, Cairo, Egypt).

Serum TNF- $\alpha$ level was determined by enzymelinked immunosorbent assay (ELISA) using
Quantikine ${ }^{\circledR}$ kit (R\&D systems, Cairo,Egypt) that contained Escherichia coli-expressed recombinant rat TNF- $\alpha$ and antibodies raised against the recombinant factor.

Total cholesterol was determined enzymatically with the cholesterol oxidase/p-aminophenazone method using Boehringer Mannheim ${ }^{\circledR}$ colorimetric kit (Mannheim, Germany). Serum was mixed in a tube containing the precipitation reagent (phosphotungstic acid and magnesium chloride) and incubated for $15 \mathrm{~min}$. After centrifugation then supernatant was mixed with reagent to measure high density cholesterol (HDL-C) content, and the clear supernatant was used for determination of its cholesterol content using method determined enzymatically with the cholesterol oxidase/paminophenazone method using Boehringer Mannheim ${ }^{\circledR} \quad$ colorimetric kit (Mannheim, Germany). Finally, LDLcholesterol was calculated according to the following Friedewald equation: total cholesterol-(HDL cholesterol+1/5 TGs) [14].

\subsection{Blood pressure measurement}

Blood pressure (BP) was measured indirectly in a conscious and slightly restrained rat by the tail cuff method as previously described $[15 ; 16]$. For these measurements,rats were conditioned to the restraint and the warming chamber for 10-20 min/day for at least 3 days before measurements. BP measurementswere performed from 7:00 to 12:00 AM by the same investigator.After 5-10 min of stabilization in a worming chamber $\left(35^{\circ} \mathrm{C}\right)$, a typical run involved 10 repetitions of the automated inflation-deflation cycle. The mean of 6 readings within a $5-10 \mathrm{mmHg}$ range wastaken as the blood pressure.

\subsection{Vascular reactivity}

Vascular reactivity was measured using the isolated artery technique as previously described $[17 ; 18]$. Thoracic aorta rings were suspended under $8 \mathrm{mN}$ resting tension in individual $30 \mathrm{ml}$ organ chambers containing Krebs-Henseleit buffer at $37^{\circ} \mathrm{C}$ and aerated with $95 \%$ oxygen, $5 \%$ carbon dioxide. Ring tension was determined by use of an isometric force transducer (Biegestab K30, HugosachsElektronik, March, Germany). Force displacement was recorded with a PowerLab Data Interface Module connected to a PC running Chart software (v4.2, ADI Instruments, Chalgrove, Oxon, UK). Rings were equilibrated for $60 \mathrm{~min}$ during which time, the bath solution was changed every 30 min. Before beginning the experiment, vessel viability was assessed by exposing arteries to $\mathrm{KCl}$ $(80 \mathrm{mM})$. This was repeated until stable responses 
were achieved (usually two exposures). For studying the vasoconstrictor responsiveness of aorta, cumulative concentrations of phenylephrine $\left(\mathrm{PE}, 10^{-9}\right.$ to $\left.10^{-5} \mathrm{M}\right)$ or $\mathrm{KCl}(10$ to $100 \mathrm{mM})$ were added to the organ bath and the response was recorded. For studying the vasodilator responsiveness of aorta, rings were first precontracted with submaximal concentrations of PE $\left(3 \times 10^{-7}\right.$ in case of diabetic group and $10^{-6}$ other groups). The submaximal concentration of PE was chosen to give similar precontraction in all studied groups. The cumulative concentrations of acetylcholine (ACh, $10^{-9}$ to $10^{-5} \mathrm{M}$ ) or sodium nitroprusside (SNP, $10^{-9}$ to $10^{-5} \mathrm{M}$ ) were then added to the organ bath and the response was recorded.

2.6Haem oxygenase-1(HO-1) immunohistochemistry

Immunohistochemistry of $\mathrm{HO}-1$ protein in rat paraffin embedded aorta sections was assessed using the method described previously [19]with some modification. The method uses primary antibody to detect the HO-1 in sections followed by fluorophore conjugated antibody.Slides were heated at $55^{\circ} \mathrm{c}$ for $30 \mathrm{~min}$. The sections were deparaffinizedin two changes of xylene (5min each) then in two changes of $100 \% \mathrm{ETOH}(5 \mathrm{~min}$ each).The sections were rehydrated through rinsing once with graded alcohols $95 \% \mathrm{ETOH}$ and $70 \% \mathrm{ETOH}$ for $2 \mathrm{~min}$ each then rinsed for $5 \mathrm{~min}$ with distilled $\mathrm{H}_{2} \mathrm{O}$. Endogenous peroxidase activity was saturate by incubating slides in $3 \% \mathrm{H} 2 \mathrm{O} 2$ solution $\left(100 \mathrm{ml} 30 \% \mathrm{H}_{2} \mathrm{O}_{2}+900 \mathrm{ml}\right.$ Methanol) for 30 min then rinsedwith 3 changesof distilled $\mathrm{H}_{2} \mathrm{O}(5$ min each). Sections were then incubated in antigen retrieval solution $(1 \mathrm{gm}$ sodium citrate in $500 \mathrm{ml}$ $\mathrm{dH}_{2} \mathrm{O}, \mathrm{pH}=6$ ) in water bath at $90^{\circ} \mathrm{c}$ for $30 \mathrm{~min}$ to unmask the antigenic epitope then the solution was allowed to cool slowly to room temperature for 20 minutes(it is important to let the temperature ramp down slowly to allow the protein molecules to fold properly). Then sections were rinsed in 3 changes of1xPBS (5 min each), 10xPBS was prepared $\left(80 \mathrm{gmNaCL}+1.94 \mathrm{gmKCL}+2.45 \mathrm{gm} \mathrm{KH} \mathrm{KH}_{2} \mathrm{PO}_{4}+\right.$ $14.2 \mathrm{gmNa}_{2} \mathrm{HPO}_{4}$ complete to $1 \mathrm{~L}$ and adjust $\mathrm{pH}$ at 7.2). Non specific binding was blocked by incubating the sections with $50 \mu \mathrm{l}$ blocking solution $(0.5 \mathrm{ml}$ rabbit serum $+9.5 \mathrm{ml} 1 \mathrm{xPBS}+100 \mu 1$ Triton $)$ for $30 \mathrm{~min}$ at room temperature.The sections were then incubated with $50 \mu \mathrm{l}$ of the rabbit anti-heme oxygenase-1 primary antibody (dilution 1:200 in blocking solution) overnight in cold room. Then sections were rinsed in 3 changesof $1 \mathrm{xPBS}(5 \mathrm{~min}$ each) then incubated with $50 \mu \mathrm{l}$ of the Alexafluor conjugated gout anti-rabbit secondary antibody (dilution 1:10,000 in blocking solution) for $2 \mathrm{hr}$ in dark then rinsed in 3 changesof $1 x \operatorname{PBS}(5 \mathrm{~min}$ each) then the coverslip was mounted with $20 \mu 1$ of fluorescence mounting media and left in dark for 20 $\mathrm{hr}$ before examination by LEICA DM500 fluorescence microscope with excitation at $\Lambda=478$ and emission at $\Lambda=495 \mathrm{~nm}$. Images were acquired with identical acquisition parameters, with minimum gain to avoid interference by tissue autofluorescence. The aorta from each rat was divided between all treatment groups. Sections treated with the secondary antibody alone did not show specific staining.

\subsection{Collagen deposition examination}

The aorta was rapidly dissected out and tissue sections $(5 \mathrm{~mm})$ fixed by immersion at room temperature in $10 \%$ neutral formalin solution. For collagen synthesis examination, paraffin-embedded tissue sections of aorta were stainedwith collagenmasson'strichrome stain. Sections were examinedunder light microscope.

2.7Intracellular of reactive oxygen species (ROS) generation

The intracellular levels of ROS were determinedby the fluorescent probedichlorofluoresceindiacetate (DCF-DA) according to the method described by [20]with slight modification. Frozen aorta were homogenized in 20 parts $(\mathrm{w} / \mathrm{v})$ of $0.32 \mathrm{~mol} / \mathrm{l}$ sucrose solution in ice and the centrifuged at $2000 \mathrm{rpm}$ at 4 ${ }^{\circ} \mathrm{Cfor} 15 \mathrm{~min}$. Then, $100 \mu \mathrm{l}$ of the supernatant was added to $1900 \mu 118: 1 \quad 40 \quad \mathrm{mmol} / \mathrm{l}$ Tris $(\mathrm{pH}$ 7.4):HEPESbuffer (10 mmol/l HEPES, $120 \mathrm{mmol} / \mathrm{l}$ $\mathrm{NaCl}, 2.5 \mathrm{mmol} / \mathrm{l} \mathrm{KCl}, 1.2 \mathrm{mmol} / \mathrm{l} \mathrm{NaH}_{2} \mathrm{PO}_{4}, 0.1$ $\mathrm{mmol} / \mathrm{l} \quad \mathrm{MgCl}_{2}, 5 \mathrm{mmol} / \mathrm{l} \quad \mathrm{NaHCO}_{3}, \quad 6 \mathrm{mmol} / \mathrm{l}$ glucose, and $1 \mathrm{mmol} / \mathrm{l} \mathrm{CaCl}_{2}$ ) containing $5 \mu \mathrm{M}$ DCF-DA plus $0.1 \%$ Pluronic F-127. The fluorescence was measured $(\lambda \mathrm{ex}=485, \lambda \mathrm{em}=515 \mathrm{~nm})$ before and after $2 \mathrm{~h}$ incubation at $37^{\circ} \mathrm{C}$. The difference in fluorescence per hour was used as indicative for ROS formation.

\subsection{Drugs and chemicals}

The following drugs and chemicals were used: STZ, ACh, PE, SNP (Sigma-Aldrich, Munich, Germany); DCF-DA, pluronic F-127 (molecular probes, Cairo, Egypt). All the used chemicals were dissolved in double distilled water.

\subsection{Statistical analysis}

All data are expressed as mean \pm SEM. Statistical analysis was performed by either student $t$ test or analysis of variance (ANOVA) followed by Newman-Keuls' post hoc test as appropriate using 
computer based fitting program and used for comparison (Prism 5, Graphpad, CA, USA).

\section{RESULTS}

\subsection{Serum parameters}

Intra-peritoneal administration of STZ in a dose of $50 \mathrm{mg} \cdot \mathrm{kg}^{-1}$ led to a significant hyperglycemia. Theelevated blood glucose levelreached maximum 3 days after STZ injection $(\mathrm{p}<0.001)$, declined a little bit (but still statistically different from control) 1 weeksafter STZ $(\mathrm{p}<0.001)$. The developed hyperglycemiawere stable in the weeks $6(\mathrm{p}<0.05)$, $8(\mathrm{p}<0.01)$ and $10(\mathrm{p}<0.05)$ after STZ injection (Fig. $1)$.

The STZ-induced hyperglycemia was associated with significant elevations in serum levels of total cholesterol $(\mathrm{p}<0.05), \quad$ LDL-cholesterol $(\mathrm{p}<0.05)$, TNF $\alpha \quad(p<0.001)$, AGEs $(p<0.01)$ and arginase activity ( $\mathrm{p}<0.001$, tables 1$)$ after 8 weeks of STZ injection.

\subsection{Blood pressure}

Diabetic animals were characterized by significantly higher systolic and diastolic BP 6 weeks after STZ injection compared with control (both at $\mathrm{p}<0.001$, Fig 2a). The elevations in systolic and diastolic BP reached steady higher levels at 8 weeks after STZ injection compared with control (both at $\mathrm{p}<0.001$, Fig 2b). However, the developed diabetes did not significantly affect the pulse (Fig 2c).

\subsection{Vascular reactivity}

Cumulative addition of $\operatorname{PE}\left(10^{-9}\right.$ to $\left.10^{-5} \mathrm{M}\right)$ or $\mathrm{KCl}$ $\left(10^{-2}\right.$ to $\left.10^{-1} \mathrm{M}\right)$ to the organ bath resulted in concentration dependent contraction of aorta in all the groups (Fig. 3). Diabetes induced by STZ resulted in larger increases in isolated aorta responsiveness to PEcompared with control. The isolated diabetic aorta hyperresponsiveness to PE started from the week 6 till the week 10 after STZ injection, reflected by a significant increase in apparent $\mathrm{E}_{\max } 6 \quad(\mathrm{p}<0.001), 8 \quad(\mathrm{p}<0.05)$ and 10 $(\mathrm{p}<0.001, \quad$ Fig 3a) weeks after STZ injection.Diabetes led also to a similar increase in the aorta responsiveness to $\mathrm{KCl}$ as shown by the significant increases in apparent $\mathrm{E}_{\max } 6,8$ and 10 (all at $p<0.001$, Fig $3 b$ ) weeks after STZ injection.

Cumulative addition of $\mathrm{ACh}\left(10^{-9}\right.$ to $\left.10^{-5} \mathrm{M}\right)$ to the organ bath resulted in concentration-related decreases in the tension of aortic artery rings precontracted with PE (Fig. 4a). Diabetes was associated with a significant decrease in isolated aorta responsiveness to ACh 8 weeks after STZ injection, reflected by a significant decrease in apparent $\mathrm{E}_{\max }(\mathrm{p}<0.05)$ compared with the control (Figure4a). On the other hand, diabetes did not show any significant effect on isolated aorta responsiveness to SNP 6, 8 or 10 weeks after STZ injection (Fig.4b).

3.4 HO-1 expression, glycogen deposition and histopathology

Aorta isolated from diabetic animals showed a clear increase in glycogen deposition 8 weeks after STZ injection compared with control (Fig 5a). However, isolated diabetic aortae did not show significant change in HO-1 immunofluorescence 8 weeks after STZ injection (Fig 5b).

3.5 Intracellular reactive oxygen species (ROS) generation

Aorta isolated from diabetic rats 8 weeks after STZ injection were characterized by a significant increase in ROS generation compared with control $(\mathrm{p}<0.05$, table 1$)$.

\section{DISCUSSION}

The purpose of this study is to fully characterize the vascular complications associated with the STZ diabetic modeland illustrate the mechanism of these vascular complications. We have shown that rats develop clear vascular complications 8 weeks after STZ injection. The developed vascular complications were characterized by hypertension, disturbed vascular reactivity, altered vascular structure and dyslipidaemia. In addition, the vascular complications were associated with low grade inflammation and oxidant stress.

In the present study, diabetes was associated with a significant increase in both systolic and diastolic BP after 6 weeks which was stable at 8 weeks. The elevated systolic (afterload) BP in diabetic animals could be due to cardiac complication and/or aortic stiffness while the elevated diastolic (preload) BP is attributed to the impaired vascular reactivity observed in diabetic animals. Aortic stiffness has been reported to be responsible for augmented pressure in late systole[21]. Previous literatures reported elevated systolic blood pressure in diabetes [22]. This elevation in systolic BP could be due to the increase inpulse and the dyslipidemia associated with diabetes. Dyslipidemia has an importanteffect on pulse in diabetes. It has been reported that increase in triglycerides andcholesterol levels were associated with increase in heart rate and are major coronaryrisk factors[23]. Furthermore, clinical trials have proved that alterationof these atherosclerotic risk factors clearly decreases cardiovascular risk [24-26]. In this study, we found significant elevations in serum levels of total cholesterol and LDL-C in diabetic animals 8 weeks after STZ injection. 
We focused on the impairment in vascular reactivity because of its importance in development of hypertension [27]. The present study has shown that aorta isolated from diabetic animals showed exaggerated contractility to $\mathrm{PE}$ and $\mathrm{KCl}$ and impaired relaxation to $\mathrm{ACh}$ compared to control after 6 weeks which were clearer at 8 weeks of STZ injection. Our result is in consistence with previous studies which have reported that diabetes is associated with enhanced vasoconstriction to different vasoconstrictors[28;29]. Diabetes-evoked vasoconstriction may be maintained by two independent mechanisms: an increased release of endothelium-derived vasoconstrictor prostanoids and an enhanced $\mathrm{Ca}^{2+}$ influx and/or sensitivity of the vascular smooth muscle cells [30]On the other hand, the impairment in endothelial dependent relaxation in diabetes is thought to be mainly mediated by inhibition of NO generation [31].

The roles of low grade inflammation in the development of vascular complications in the present diabetic model were investigated by monitoring serum levels of TNF- $\alpha$ (one of the important inflammatory cytokine), arginase enzyme (an important enzyme in vascular inflammation), aortic ROS generation and HO-1 (homeostatic enzyme) expression. In the present study, we found significant increases in serum levels of TNF- $\alpha$, arginase enzyme, and aortic ROS generation in diabetic animals 8 weeks after STZ injection. Plasma levels of TNF- $\alpha$ were found to increase in patients with both type 1 and type 2 diabetes and are significantly correlated to elevated fasting glucose levels, it has also been suggested to represent an important link between metabolic deregulations and inflammation in diabetes [32].Elevated ROS is linked to the formation of AGEs during prolonged hyperglycaemia in diabetes as both hyperglycaemia and oxidative stress in diabetes promote AGEs accumulation[33].AGEs and their intermediates have been found to contribute to vascular complications associated with diabetes and increased oxidative stress [34].In the present work we have reported a significant increase in serum level of AGEs in diabetic animals. Superoxide, one of the ROS produced mainly by NADPH oxidase and increased in diabetes, directly decreases NO bioavailability by reacting with $\mathrm{NO}$, forming peroxynitrite at a rate up to four times faster than superoxide metabolism by superoxide dismutase [35]. During diabetes, impaired vascular function is closely associated with oxidative stress and vascular inflammation [36], both of which have been associated with increases in arginase activity and expression [37]. Arginase, which L-arginine to urea and ornithine, competes directly with NOS for Larginine. Hence increases in arginase activity can decrease tissue and cellular arginine levels, reducing its availability to eNOS[38]. This may lead to decreased NO production and increased production of superoxide by eNOS[39]. We examined HO-1 expression as one of the most important homeostatic mechanisms. Expression of HO-1 prevents the development of atherosclerotic lesions[40], lowers blood pressure[41]and prevents vascular dysfunction[42]in a variety of experimental conditions. In the present study, we did not notice any major change in HO-1 expression in aorta isolated from diabetic animals compared with control.

The structural changes in the vasculature were also investigated in the present study. We found a clear increase in collagen deposition in aorta isolated from diabetic animals compared with control. Collagen is the major biomechanical constituent of the vessel wall;pathological modifications in fibrous collagen might alter the wall stiffness and consequently derange vascular function(Kesava, 2004). Collagen in the blood vessel wall, with time and under the effect of AGEs, undergoes significant nonenzymic glycosylation (glycation), which may have a considerable bearing on the atherosclerotic process, especially the accelerated atherogenesis associated with diabetes [43-45].

\section{CONCLUSION}

In conclusion, single dose of $50 \mathrm{mg} \cdot \mathrm{kg}^{-1}$ of STZ produces a model of diabetic vascular complication after 8 weeks that are characterized by hypertension, disturbed vascular reactivity, increased serum lipids, inflammatory cytokines and enzymes, ROS generation and aortic collagen deposition.

\section{ACKNOWLEDGMENT}

This work is funded by a research grant ID1024 provided by the Science and Technology Development Fund, Egypt.

\section{REFERENCE}

1. Shantikumar S, Caporali A, Emanueli C, Role of microRNAs in diabetes and its cardiovascular complications. Cardiovasc Res 2011;cvr300.

2. Butler R, Macdonald T, Struthers AD, Morris AD, The clinical implications of diabetic heart disease. Eur Heart J 1998;19:1617-27.

3. Marks JB and Raskin P, Cardiovascular risk in diabetes: a brief review. J Diabetes Complications 2000;14:108-15. 
4. Brownlee M, Biochemistry and molecular cell biology of diabetic complications. Nature 2001;414:813-20.

5. Guzik TJ, Mussa S, Gastaldi D, Sadowski J, Ratnatunga C, Pillai R, Channon KM, Mechanisms of Increased Vascular Superoxide Production in Human Diabetes Mellitus: Role of $\mathrm{NAD}(\mathrm{P}) \mathrm{H}$ Oxidase and Endothelial Nitric Oxide Synthase. Circulation 2002;105:1656-62.

6. Senses V, Ozyazgan S, Ince E, Tuncdemir M, Kaya F, Ozturk M, Sultuybek G, Akkan AG, Effect of 5aminoimidazole-4-carboxamide riboside (AICA-r) on isolated thoracic aorta responses in streptozotocin-diabetic rats. J Basic Clin Physiol Pharmacol 2001;12:227-48.

7. Kamata K, Miyata N, Kasuya Y, Impairment of endothelium-dependent relaxation and changes in levels of cyclic GMP in aorta from streptozotocininduced diabetic rats. Br J Pharmacol 1989;97:6148.

8. Akbari CM and LoGerfo FW, Diabetes and peripheral vascular disease. J Vasc Surg 1999;30:373-84.

9. Szkudelski T, Streptozotocin-nicotinamide-induced diabetes in the rat. Characteristics of the experimental model. Exp Biol Med 2012;237:48190.

10. Bimenya GS, Nzarubara GR, Kiconco J, Sabuni S, Byarugaba $\mathrm{W}$, The accuracy of self monitoring blood glucose meter systems in Kampala Uganda. Afr Health Sci 2003;3:23-32.

11. El-Bassossy HM, El-Fawal R, Fahmy A, Arginase inhibition alleviates hypertension associated with diabetes: Effect on endothelial dependent relaxation and NO production. Vascul Pharmacol 2012;57:194-200.

12. El-Bassossy HM, Abo-Warda SM, Fahmy A, Rosiglitazone, a peroxisome proliferator-activated receptor $\mathrm{g}$ stimulant, abrogates diabetes-evoked hypertension by rectifying abnormalities in vascular reactivity. Clin Exp Pharmacol Physiol 2012;39:643-9.

13. Sampathkumar R, Balasubramanyam M, Rema M, Premanand C, Mohan V, A novel advanced glycation index and its association with diabetes and microangiopathy. Metabolism 2005;54:1002-7.

14. Fukuyama N, Homma K, Wakana N, Kudo K, Suyama A, Ohazama H, Tsuji C, Ishiwata K, Eguchi Y, Nakazawa H, Tanaka E, Validation of the Friedewald Equation for Evaluation of Plasma LDL-Cholesterol. J Clin Biochem Nutr 2008;43:15.

15. Badawy D, El-Bassossy HM, Fahmy A, Azhar A, Aldose reductase inhibitors, zopolrestat and ferulic acid, alleviate hypertension associated with diabetes: Effect on vascular reactivity. Can J Physiol Pharmacol 2012; In press.
16. El-Bassossy HM, Fahmy A, Badawy D, Cinnamaldehyde protects from the hypertension associated with diabetes. Food Chem Toxicol 2011;49:3007-12.

17. El-Bassossy HM, El-Moselhy MA, Mahmoud MF, Pentoxifylline alleviates vascular impairment in insulin resistance via TNF-? inhibition. Naunyn Schmiedebergs Arch Pharmacol 2011;384:277-85.

18. Mahmoud MF, El-Nagar M, El-Bassossy HM, Antiinflammatory effect of atorvastatin on vascular reactivity and insulin resistance in fructose fed rats. Arch Pharm Res 2012;35:155-62.

19. El-Bassossy HM, El-Maraghy NN, El-Fayoumi HM, Watson ML, Haem oxygenase-1 induction protects against tumour necrosis factor alpha impairment of endothelial-dependent relaxation in rat isolated pulmonary artery. $\mathrm{Br} \mathrm{J}$ Pharmacol 2009;158:1527-35.

20. Ugochukwu NH and Cobourne MK, Modification of renal oxidative stress and lipid peroxidation in streptozotocin-induced diabetic rats treated with extracts from Gongronema latifolium leaves. Clin Chim Acta 2003;336:73-81.

21. O'Rourke MF and Nichols WW, Aortic Diameter, Aortic Stiffness, and Wave Reflection Increase With Age and Isolated Systolic Hypertension. Hypertension 2005;45:652-8.

22. Majithiya JB and Balaraman R, Metformin reduces blood pressure and restores endothelial function in aorta of streptozotocin-induced diabetic rats. Life Sciences 2006;78:2615-24.

23. Eliza J, Daisy P, Ignacimuthu S, Duraipandiyan V, Antidiabetic and antilipidemic effect of eremanthin from Costus speciosus (Koen.)Sm., in STZ-induced diabetic rats. Chem Biol Interact 2009;182:67-72.

24. Pedersen TR, Coronary artery disease: the Scandinavian Simvastatin Survival Study experience. Am J Cardiol 1998;82:53T-6T.

25. Robins SJ, Targeting low high-density lipoprotein cholesterol for therapy: lessons from the Veterans Affairs High-density Lipoprotein Intervention Trial. Am J Cardiol 2001;88:19N-23N.

26. Zanchetti A, Hansson L, Dahlof B, Elmfeldt D, Kjeldsen S, Kolloch R, Larochelle P, McInnes GT, Mallion JM, Ruilope L, Wedel $\mathrm{H}$, Effects of individual risk factors on the incidence of cardiovascular events in the treated hypertensive patients of the Hypertension Optimal Treatment Study. HOT Study Group. J Hypertens 2001;19:1149-59.

27. Mansour SM, Bahgat AK, El-Khatib AS, Khayyal MT, Ginkgo biloba extract (EGb 761) normalizes hypertension in $2 \mathrm{~K}, 1 \mathrm{C}$ hypertensive rats: role of antioxidant mechanisms, ACE inhibiting activity and improvement of endothelial dysfunction. Phytomedicine 2011;18:641-7.

28. El-Bassossy HM, Fahmy A, Badawy D, Cinnamaldehyde protects from the hypertension 
associated with diabetes. Food and Chemical Toxicology 2011;49:3007-12.

29. Kinoshita Y, Saito M, Satoh I, Shomori K, Suzuki H, Yamada M, Kono T, Satoh K, General administration of cyclohexenonic long-chain fatty alcohol ameliorates hyperreactivity of STZ-induced diabetic rat aorta. Life Sciences 2006;78:1508-14.

30. Xavier FE, Davel AP, Rossoni LV, Vassallo DV, Time-dependent hyperreactivity to phenylephrine in aorta from untreated diabetic rats: role of prostanoids and calcium mobilization. Vascular Pharmacology 2003;40:67-76.

31. El-Bassossy HM, El-Moselhy MA, Mahmoud MF, Pentoxifylline alleviates vascular impairment in insulin resistance via TNF-? inhibition. Naunyn Schmiedebergs Arch Pharmacol 2011;384:277-85.

32. Nilsson-Ohman J, Fredrikson GN, NilssonBerglund LM, Gustavsson C, Bengtsson E, Smith ML, Agardh CD, Agardh E, Jovinge S, Gomez MF, Nilsson J, Tumor Necrosis Factor-\{alpha $\}$ Does Not Mediate Diabetes-Induced Vascular Inflammation in Mice. Arterioscler Thromb Vasc Biol 2009;29:1465-70.

33. Aronson D, Hyperglycemia and the pathobiology of diabetic complications. Adv Cardiol 2008;45:1-16.

34. He M, Siow RCM, Sugden D, Gao L, Cheng X, Mann GE, Induction of HO-1 and redox signaling in endothelial cells by advanced glycation end products: A role for Nrf2 in vascular protection in diabetes. Nutrition, Metabolism and Cardiovascular Diseases 2011;21:277-85.

35. Beckman JS, Oxidative damage and tyrosine nitration from peroxynitrite. Chem Res Toxicol 1996;9:836-44.

36. El-Bassossy HM, El-Moselhy MA, Mahmoud MF, Pentoxifylline alleviates vascular impairment in insulin resistance via TNF-? inhibition. Naunyn Schmiedebergs Arch Pharmacol 2011;384:277-85.
37. Bivalacqua TJ, Hellstrom WJ, Kadowitz PJ, Champion $\mathrm{HC}$, Increased expression of arginase II in human diabetic corpus cavernosum: in diabeticassociated erectile dysfunction. Biochem Biophys Res Commun 2001;283:923-7.

38. Romero MJ, Platt DH, Tawfik HE, Labazi M, ElRemessy AB, Bartoli M, Caldwell RB, Caldwell RW, Diabetes-induced Coronary Vascular Dysfunction Involves Increased Arginase Activity. Circ Res 2008;102:95-102.

39. Berkowitz DE, White R, Li D, Minhas KM, Cernetich A, Kim S, Burke S, Shoukas AA, Nyhan D, Champion HC, Hare JM, Arginase Reciprocally Regulates Nitric Oxide Synthase Activity and Contributes to Endothelial Dysfunction in Aging Blood Vessels. Circulation 2003;108:2000-6.

40. Pombo Gregoire I, Correia AM, Soares MP, TuP7:62 Heme oxygenase-1 derived carbon monoxide inhibits atherosclerosis. Atherosclerosis Supplements 2006;7:198.

41. Cao J, Inoue K, Li X, Drummond G, Abraham NG, Physiological significance of heme oxygenase in hypertension. Int $\mathrm{J}$ Biochem Cell Biol 2009;41:1025-33.

42. Abraham NG and Kappas A, Pharmacological and Clinical Aspects of Heme Oxygenase. Pharmacol Rev 2008;60:79-127.

43. Bailey AJ, Paul RG, Knott L, Mechanisms of maturation and ageing of collagen. Mech Ageing Dev 1998;106:1-56.

44. Barnes MJ and Farndale RW, Collagens and atherosclerosis. Exp Gerontol 1999;34:513-25.

45. Chappey O, Dosquet C, Wautier MP, Wautier JL, Advanced glycation end products, oxidant stress and vascular lesions. Eur J Clin Invest 1997;27:97108. 